\title{
A proposed SyS Case Definition for Opioid Overdose Related ED visits- an evaluation in three regions
}

\author{
Yushiuan Chen², Sandra Gonzalez ${ }^{3,4}$, Harold Gili5, Michele Askenazi', \\ Felicia Quintana-Zinn ${ }^{3}$, Arthur Davidson ${ }^{6}$, Christine Billings ${ }^{7}$ and Ming $\mathbf{Q u}^{3}$
}

\begin{abstract}
${ }^{1}$ Tri-County Health Department, Greenwood Village, CO, USA; ${ }^{2}$ Tri-County Health Department, Greenwood Village, CO, USA; ${ }^{3}$ Nebraska Department of Health and Human Services, Lincoln, NE, USA; ${ }^{4}$ University of Nebraska-Lincoln, Lincoln, NE, USA; ${ }^{5}$ Marion County Public Health Department, Marion County, CO, USA; ${ }^{6}$ Denver Public Health, Denver, CO, USA; ${ }^{7}$ Jefferson County Public Health, Golden, CO, USA
\end{abstract}

\section{Objective}

The objective is to develop a standard opioid overdose case definition that could be generalized nationally.

\section{Introduction}

Opioid ODs have been rising globally and nationally. The death rate from ODs in the United States has increased 137\% since 2000, including a $200 \%$ increase of OD deaths involving opioids ${ }^{1}$. The pilot project, a collaboration across 3 states, allowed information sharing with Syndromic surveillance (SyS) partners across jurisdictions, such as sharing a standard SyS case definition and verifying its applicability in each jurisdiction. This is a continuation of the work from an initial pilot project presented during the ISDS Opioid OD Webinar series.

\section{Methods}

Three regions (Colorado North Central Region [CO-NCR]), State of Nebraska [NE], and Marion County, Indiana) participated in the development and evaluation of the opioid OD case definition. Data sources included ESSENCE and 2015 hospital discharge data (HDD) for the first two jurisdictions. Work was conducted in 3 stages. Stage I and II consisted of the development and validation of an opioid misuse definition. In stage I, the percent of completeness of admission date, chief complaint (CC), and discharge diagnosis (DD) was assessed from January 2015 to August 2016 SyS emergency department (ED) data from each of the 3 participating jurisdictions. Data selected for the time period with the best completeness among all jurisdicions was utilized to develop a case definition. Completeness of ESSENCE data submission was assessed at all jurisdicions. The threshold for best data quality was $80 \%$ of completeness. SyS ED data was analyzed for the selection of CC search terms and ICD9/ICD10² DD codes, and the reported Chief Complaint-Discharge Diagnosis (CCDD) were validated by analyzing consistency between $\mathrm{CC}$ and DD. In stage II, the consistency of DD reporting corresponding to the opioid case definition was assessed for CO-NCR and NE data by performing Pearson Correlation analysis to compare the weekly counts of opioid misuse cases in 2015 SyS ED data to those obtained in HDD. Stage III consisted of the development of an opioid OD case definition that meets the DD code reporting requirements of the Centers for Disease Control and Prevention (CDC), Prescription Drug Overdose Prevention for States awardees. This definition consisted of an ESSENCE query containing CC, and CCDD components. For Stage III, SyS ED data was analyzed for the August 2016 to August 2017 time period. The case definition was evaluated by assessing the consistency between the CC and DD reported for each identified opioid OD possible case. Triage notes were used for case validation.

\section{Results}

Stage I: Mean percent of completeness of DD codes for CO-NCR, NE and Marion County, IN, 2015 ED SyS data was $\geq 85 \%$. In the CO-NCR, of 963 cases detected by the CC definition, $99.4 \%$ had an opioid misuse diagnostic code in the $\mathrm{DD}$, while of 1,445 cases detected by the DD, $66.2 \%$ had an associated opioid misuse in the
$\mathrm{CC}$ search terms. In NE, of 6 cases detected by the $\mathrm{CC}$ definition, $33 \%$ identified opioid misuse DD. However, of 42 cases detected by the DD definition, only 5\% identified opioid misuse CC search terms. In Marion County, IN, of 95 cases detected by the $\mathrm{CC}$ definition, $70 \%$ identified opioid related diagnosis codes. Of 191 cases detected by the DD definition, only $20 \%$ identified opioid-related CC search terms. Stage II: Results of the Pearson correlation analysis indicate statistically significant correlations between 2015 SyS and HDD data for the DD code based opioid definition for both $\mathrm{CO}(\mathrm{r}=0.92, \mathrm{p}<$ $0.0001)$, and NE $(r=0.63, p<0.0001)$. Stage III: In NE, $56 \%$ of the cases detected by the CC component, identified opioid OD DD codes, and only $8 \%$ of the cases detected by the DD component identified opioid OD search terms in the CC. Triage notes were consistent with opioid OD in $55 \%$ of the cases detected by the DD component. However, for CO-NCR, of 235 cases detected by the CC component, 215 identified opioid OD DD codes. Of 465 cases detected by the DD component, $46 \%$ identified opioid OD search terms in the CC field. Triage notes values were consistent with opioid OD reported DD codes in $80 \%$ of the cases.

\section{Conclusions}

Results suggest that DD codes reported in SyS ED data correlated with HDD. Indicators of opioid OD signs and symptoms were observed in CCDD. Therefore, the SyS case definition proposed through this pilot project may be applied by other states to support real-time monitoring of opioid OD related hospital ED visits, and consequences of opioid OD. Further study includes exploring how triage notes search terms may improve the identification of opioid OD related ED visits.

\section{Keywords}

opioid overdose; emergency department; drug/substance misuse; syndromic surveillance; ESSENCE

\section{Acknowledgments}

Boulder County Public Health (Tailia Brown), Denver Public Health (Cali Zimmerman), Jefferson County Public Health (Angel Anderson and Kate Watkins), Hospitals, CDC NSSP, Johns Hopkins University ESSENCE

\section{References}

1. Rudd, R. A., Aleshire, N., Zibbell, J. E., \& Gladden, R. M. (2016). Increases in drug and Opioid overdose deaths - United States, 20002014. MMWR. Morbidity and Mortality Weekly Report, 64(50-51), 1378-1382.

2. Ising, A., Proescholdbell, S., Harmon, K. J., Sachdeva, N., Marshall, S. W., \& Waller, A. E. (2016). Use of syndromic surveillance data to monitor poisonings and drug overdoses in state and local public health agencies. Injury Prevention, 22(Suppl 1), i43-i49.

\section{*Yushiuan Chen \\ E-mail: ychen@tchd.org}

